Louisiana State University

LSU Digital Commons

Faculty Publications

Department of Physics \& Astronomy

$1-1-1999$

\title{
Temporal coherence of high-order harmonics
}

C. Lyngå

Lunds Tekniska Högskola

M. B. Gaarde

Lunds Tekniska Högskola

C. Delfin

Lunds Tekniska Högskola

M. Bellini

LENS - European Laboratory for Non-Linear Spectroscopy

T. W. Hänsch

Istituto Nazionale per la Fisica della Materia

See next page for additional authors

Follow this and additional works at: https://digitalcommons.Isu.edu/physics_astronomy_pubs

\section{Recommended Citation}

Lyngå, C., Gaarde, M., Delfın, C., Bellini, M., Hänsch, T., L' Huillier, A., \& Wahlström, C. (1999). Temporal coherence of high-order harmonics. Physical Review A - Atomic, Molecular, and Optical Physics, 60 (6), 4823-4830. https://doi.org/10.1103/PhysRevA.60.4823

This Article is brought to you for free and open access by the Department of Physics \& Astronomy at LSU Digital Commons. It has been accepted for inclusion in Faculty Publications by an authorized administrator of LSU Digital Commons. For more information, please contact ir@lsu.edu. 


\section{Authors}

C. Lyngå, M. B. Gaarde, C. Delfın, M. Bellini, T. W. Hänsch, A. L' Huillier, and C. G. Wahlström 


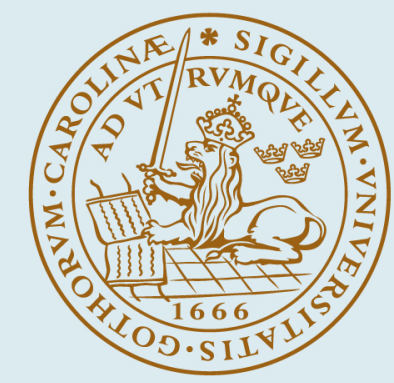

\title{
LUND UNIVERSITY
}

\section{Temporal coherence of high-order harmonics}

\author{
Lynga, C; Gaarde, Mette; Delfin, C; Bellini, M; Hansch, T. W; L'Huillier, Anne; Wahlström, \\ Claes-Göran \\ Published in: \\ Physical Review A (Atomic, Molecular and Optical Physics)
}

DOI:

10.1103/PhysRevA.60.4823

1999

Link to publication

Citation for published version (APA):

Lynga, C., Gaarde, M., Delfin, C., Bellini, M., Hansch, T. W., L'Huillier, A., \& Wahlström, C-G. (1999). Temporal coherence of high-order harmonics. Physical Review A (Atomic, Molecular and Optical Physics), 60(6), 48234830. https://doi.org/10.1103/PhysRevA.60.4823

\section{Total number of authors:}

7

\section{General rights}

Unless other specific re-use rights are stated the following general rights apply:

Copyright and moral rights for the publications made accessible in the public portal are retained by the authors and/or other copyright owners and it is a condition of accessing publications that users recognise and abide by the legal requirements associated with these rights.

- Users may download and print one copy of any publication from the public portal for the purpose of private study or research.

- You may not further distribute the material or use it for any profit-making activity or commercial gain

- You may freely distribute the URL identifying the publication in the public portal

Read more about Creative commons licenses: https://creativecommons.org/licenses/

Take down policy

If you believe that this document breaches copyright please contact us providing details, and we will remove

access to the work immediately and investigate your claim.
} 


\title{
Temporal coherence of high-order harmonics
}

\author{
C. Lyngå, ${ }^{1}$ M. B. Gaarde,,${ }^{1,2}$ C. Delfin, ${ }^{1}$ M. Bellini, ${ }^{3,4}$ T. W. Hänsch, ${ }^{4,5,6}$ A. L' Huillier, ${ }^{1}$ and C.-G. Wahlström ${ }^{1}$ \\ ${ }^{1}$ Department of Physics, Lund Institute of Technology, P.O. Box 118, S-221 00 Lund, Sweden \\ ${ }^{2}$ Niels Bohr Institute, Orsted Laboratory, Universitetsparken 5, 2100 Copenhagen, Denmark \\ ${ }^{3}$ European Laboratory for Non Linear Spectroscopy (LENS), Largo Enrico Fermi, 2, I-50125 Florence, Italy \\ ${ }^{4}$ Istituto Nazionale di Fisica della Materia (INFM), Italy \\ ${ }^{5}$ Department of Physics, University of Florence, Largo Enrico Fermi, 2, I-50125 Florence, Italy \\ ${ }^{6}$ Max-Planck-Institut für Quantenoptik, P.O. Box 1513, D-85740 Garching, Germany
}

(Received 1 June 1999)

\begin{abstract}
Systematic studies of the temporal coherence properties of high-order harmonic radiation are presented. These complement our previous investigations [Bellini et al., Phys. Rev. Lett. 81, 297 (1998)], where we showed the separation of the far-field pattern of high-order harmonics into two distinct spatial regions with different coherence times. Here we show how the coherence time of the inner and outer regions changes as a function of the harmonic order, the laser intensity, and the focusing conditions. Good agreement with the predictions of the semiclassical model of harmonic generation is obtained. [S1050-2947(99)10312-3]
\end{abstract}

PACS number(s): 32.80.Rm, 42.65.Ky

\section{INTRODUCTION}

High-order harmonic generation has been studied extensively during the past years. A good understanding has now been reached of the fundamental processes behind harmonic generation, and the harmonic radiation has been characterized through systematic theoretical and experimental studies. Important properties like the pulse length [1,2] and the spatial coherence [3] have been investigated. The harmonic radiation presents a unique source in the extreme ultraviolet (XUV) spectral region, and has recently been used in several applications [4]. A characterization of the coherence properties of the source is required for applications such as interferometry and spectroscopy in the XUV region. In addition, coherence properties are of fundamental interest for the study of the dynamics of the harmonic generation process, since, for instance, the temporal coherence gives information about the time dependence of the phase of the harmonic radiation.

In a previous experiment [5], we measured the temporal coherence of high-order harmonic radiation created with 100-fs titanium-sapphire (Ti:S) laser pulses. This was done by studying the far-field interference fringes of harmonic radiation produced in two separate sources, originating from the same laser pulse, and therefore phase locked [6]. The coherence time was measured by varying the time delay between the two pulses generating the two harmonic sources and recording the decrease of contrast in the fringe pattern. Very interestingly, we found that the far-field pattern consists of two distinct spatial regions with different coherence times. The inner (central) region has a long coherence time, comparable to the estimated duration of the harmonic pulse, while the outer region has a much shorter coherence time, of a few femtoseconds. Our interpretation of the experimental results [5] is based on a semiclassical model for harmonic generation [7-9]. (A more detailed theoretical analysis was also presented in Ref. [10].)

Briefly, the semiclassical model assumes that an atom interacting with an intense laser field can be described as having only one active electron and only one bound state. The electron, experiencing the sum of the Coulomb field and the intense electromagnetic laser field, can tunnel through the barrier formed by the resulting potential, and subsequently be accelerated by the laser field. For a linearly polarized laser field, the electron may be driven back toward the core and recombine. When decaying to the ground state, it gives rise to the emission of a high-energy photon. For the generation of some particular harmonic there are several possible trajectories such that the electron returns to the core with the correct energy, depending on the times of release from and return to the core. In Ref. [11] it was shown that for a harmonic belonging to the plateau region, there are two trajectories that dominate the generation process. The phase of the harmonic dipole moment is related to that of the electronic wave function, and is classically equal to the action of the trajectory of the laser-driven returning electron. In particular, it is proportional to the intensity of the driving field. The proportionality factor, or phase coefficient $\alpha$, is closely related to the excursion time ${ }^{1} \tau$, the amount of time the electron spends in the continuum. The two trajectories, having different excursion times, therefore contribute to the dipole moment with different phase dependencies as a function of the laser intensity.

The interpretation of the experimental results presented in Ref. [5] is based on the above result, namely, that for each harmonic $q$ there are mainly two contributions to the dipole moment at the harmonic frequency. Each of these components has a phase that is proportional to the intensity, and we can thus write the amplitude of the dipole moment of the $q$ th harmonic approximately as

$$
d_{q}(I)=A_{1}(I) \exp \left(-i \alpha_{1} I\right)+A_{2}(I) \exp \left(-i \alpha_{2} I\right)
$$

where $A_{j}(I)$ is the strength of the component corresponding to the trajectory $j$, and $\alpha_{j}$ is the phase coefficient. Since the intensity varies both in time and space, the harmonic radiation field consists of two components with different temporal

\footnotetext{
${ }^{1}$ In previous papers the excursion time was referred to as the return time.
} 


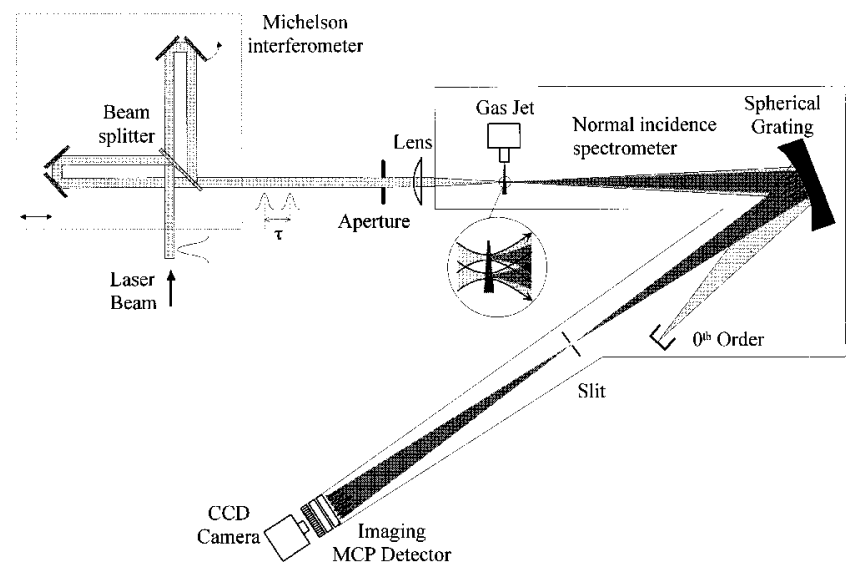

FIG. 1. Experimental setup using the normal-incidence spectrometer.

and spatial phase behaviors. The spatial variation with intensity gives rise to a curvature of the phase front, and thereby a different divergence for each field component. This results in a complex far-field pattern for the total field, with two regions, as observed in the experiment [5]. Likewise, the temporal variation induces a time-dependent frequency variation (a chirp), different for each field component. This leads to different coherence times for the two spatial regions. The results presented in Ref. [5] are probably some of the clearest experimental evidence for the semiclassical interpretation of harmonic generation, since their interpretation involves the existence of several contributing and interfering trajectories. However, they were obtained at a constant laser intensity and only for a few low-order harmonics (up to the 15 th harmonic). Measurements of the two coherence times for the two spatial regions of the far-field pattern were performed only for one harmonic, the 15th, generated in argon.

The present work is a follow up of the experiment presented in Ref. [5]. We extend our previous experiment, and perform more systematic experimental studies, as well as a more detailed theoretical analysis. The coherence times of the inner and outer regions in the far-field profile are studied as a function of several parameters: harmonic order, laser intensity, and geometrical conditions (position of the gas jet relative to the laser focus). We show how the coherence times vary as a function of these different parameters, and we compare the results with the predictions of the semiclassical model.
In Sec. II, we describe the experimental setup and the method of analysis which we have employed to determine the coherence times. Our theoretical model is presented in Sec. III, and the main results of this work are presented in Sec. IV. We summarize in Sec. V.

\section{EXPERIMENTAL SETUP AND METHOD}

The laser used is the terawatt laser of the Lund HighPower Laser Facility [12], which is based on chirped pulse amplification in titanium-doped sapphire. The laser operates at $10 \mathrm{~Hz}$, and produces pulses with an energy of up to 200 $\mathrm{mJ}$ in a beam of about $5-\mathrm{cm}$ diameter. The wavelength is 800 $\mathrm{nm}$ and the pulse length around $110 \mathrm{fs}$.

The experimental setup is shown in Fig. 1. It presents large similarities with the one used in our previous experiment [5]. A Michelson interferometer separates the laser pulse into two identical pulses with a variable time delay between them. The time delay is controlled by manually translating one pair of mirrors (see Fig. 1). One mirror is slightly tilted so that the two beams are not completely parallel. The beams are focused by a lens placed after the interferometer into an argon gas jet. Since the two beams are not exactly parallel they are focused at two slightly different positions, and we produce two sources of harmonics at a close distance but spatially separated. The harmonic beams from the two sources are practically superposed in the far field, giving rise to an interference pattern. A grating is used to select a given harmonic order and project it onto a microchannel plate, coupled to a phosphor screen. The image on the phosphor screen is captured with a CCD camera. All the images presented in this paper are single-shot recordings.

The fringe separation in the far field is given by $\Delta y$ $=L \lambda / \delta$, just as in a Young's double-slit experiment, where $L$ is the distance between the gas jet (or the image of the gas jet) and the detection plane where the interference pattern is recorded, $\lambda$ is the harmonic wavelength, and $\delta$ is the distance between the two sources. A smaller distance between the two foci thus gives a larger fringe separation. For a given focusing geometry (and more exactly for a given numerical aperture, or $f$ ), the smallest distance between the two foci cannot be less than the beam waist diameter. Below that distance, the two fundamental beams interfere and the two harmonic sources cannot be considered as independent. Consequently, for high harmonic orders the focusing geometry has to be chosen to be relatively tight, and the two foci located as
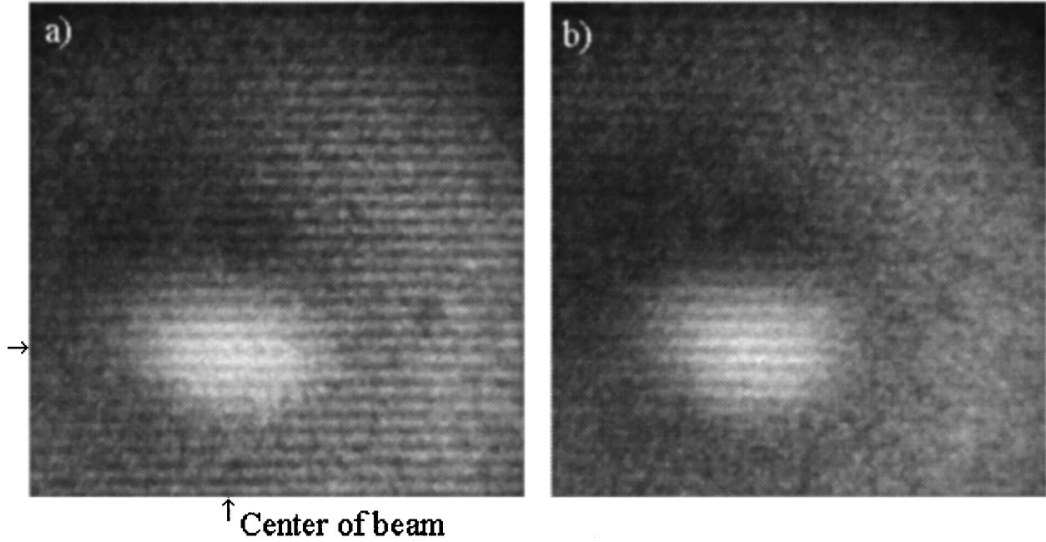

FIG. 2. Interference pattern for the 13th harmonic using a delay between pulses of (a) $\tau=0$ fs and (b) $\tau=25$ fs. 
close as possible to be able to resolve the fringes. This is to the detriment, however, of the harmonic yield. Depending on the focusing geometry we typically work with a separation between the foci of $100-150 \mu \mathrm{m}$. The laser beams are apertured to a diameter of $9 \mathrm{~mm}(f=28)$, except for the results presented in Figs. 2 and 10 where an aperture of $6 \mathrm{~mm}(f$ $=42$ ) is used. Then they are focused by a $25-\mathrm{cm}$ lens. We estimate the spot sizes to be approximately 80 and $120 \mu \mathrm{m}$, respectively. The spot size is taken as the diameter of the Airy disk formed when focusing a top hat intensity function (estimating the laser to be 1.5 times diffraction limited). Depending on the laser energy and the size of the aperture, the peak laser intensity in each of the two foci is between 1 $\times 10^{14}$ and $5 \times 10^{14} \mathrm{~W} / \mathrm{cm}^{2}$.

The optical quality and mechanical stability of the Michelson interferometer is critical to obtain accurate and reproducible data. The dielectric mirrors (50-mm diameter) are of $\lambda / 10$ flatness. The beam splitter $\left(60 \times 80 \mathrm{~mm}^{2}\right)$ is chosen to be rather thin $(3 \mathrm{~mm})$ in order to limit the influence of nonlinear effects. Probably as a consequence of the small thickness, the beam splitter used in our setup, in spite of the $\lambda / 10$ flatness requirement, presents a curvature. This leads to slightly different divergences in the two beams after reflections on opposite sides of the beam splitter. The two foci are therefore not strictly in the same plane. The minimization of this effect is another reason for us to use a relatively tight focusing geometry.

Working in the above mentioned intensity region means that we are around the saturation intensity for argon (about $3 \times 10^{14} \mathrm{~W} / \mathrm{cm}^{2}$ ). We perform measurements in argon up to the 31st harmonic order, which corresponds to the cutoff region where the harmonic intensity decreases rapidly with the process order. We also observe interference fringes in neon. However, due to the tight focusing geometry the harmonic yield is too low for systematic studies.

Two different setups are used for the spectral analysis of the harmonic radiation. For the lower-order harmonics (13th-23rd) we use a normal incidence spectrometer with a spherical grating (1200 lines $/ \mathrm{mm})$. The normal-incidence spectrometer (shown in the figure) is well suited for an analysis of lower-order harmonics where a large number of photons are available, but for high-order harmonics the reflectivity of the grating is too low (typically $2-3 \%$ ). For the studies of the 17 th-31st harmonics, we use a grazingincidence toroidal grating with 700 lines $/ \mathrm{mm}$ and a reflectivity of about $10 \%$. This allows us to collect a larger number of photons, but to the detriment of the spatial profile. Indeed, astigmatism and other aberrations are more severe with the use of a grazing incidence grating.

Figure 2 shows the far-field pattern of the 13th harmonic for two different time delays, $\tau$, between the pulses (a) 0 fs and (b) 25 fs. The peak intensity is approximately 3 $\times 10^{14} \mathrm{~W} / \mathrm{cm}^{2}$. Two distinct spatial regions can be seen, an inner intense region and an outer less intense region. Fringes exist in both regions in (a) while the fringes in the outer region have disappeared in (b), demonstrating that the coherence time in the inner region is longer than in the outer. This confirms and generalizes the results of Ref. [5], since the same effect is observed here at a higher laser intensity, with a different setup and laser system, and for a different harmonic order.
The experimental studies presented below consist in measuring the coherence time in different regions of the spatial profile as a function of several parameters. The coherence time is equal to the half-width at half-maximum of the curve $V(\tau)$ representing the visibility of the fringes in some spatial region as a function of the delay between the two pulses [13]. The visibility is defined by the ratio $V=\left(I_{\max }\right.$ $\left.-I_{\min }\right) /\left(I_{\max }+I_{\min }\right)$, where $I_{\max }\left(I_{\min }\right)$ is the maximum (minimum) intensity in the spatial region considered. The visibility is theoretically equal to 1 when the two pulses are temporally overlapped and have equal intensity, and decreases as a function of the time delay $\tau$. To determine experimentally the visibility curve $V(\tau)$, we record several images at different time delays. For each image we calculate the visibility in a given spatial region and thereby determine the (local) coherence time by plotting $V(\tau)$. This procedure is very time consuming. Since the aim of this work is to study the variation of the coherence time as a function of different parameters and to compare the results with the predictions of the semiclassical model, we have concentrated on studying trends rather than accurately determining absolute coherence times. Therefore, for the systematic studies presented below, we use an alternative, more approximate, method. It consists of recording the time delays at which our eye stops or begins seeing interference fringes. The time span over which the fringes are visible to the eye is then calibrated against the coherence time measured by recording a visibility curve. We find that our "eye's visibility time" corresponds to the coherence time multiplied by a factor of 3.7. Below we refer to this eye's visibility time, divided by the factor 3.7 , as the experimental coherence time.

\section{THEORETICAL BACKGROUND}

We first recall some elementary definitions and results related to the temporal coherence properties of an electromagnetic field, before applying them to the particular case of harmonic generation.

\section{A. Definition of the coherence time}

The temporal coherence is characterized theoretically by the first-order correlation function

$$
\Gamma^{(1)}(\vec{r}, \tau)=\lim _{T \rightarrow \infty} \frac{1}{T} \int_{0}^{T} \mathcal{E}(\vec{r}, t+\tau) \mathcal{E}^{*}(\vec{r}, t) d t,
$$

where $\mathcal{E}$ denotes the electromagnetic field. The modulus of the normalized correlation function (also called the degree of temporal coherence) coincides with the visibility function introduced above, which can be measured experimentally. Its half-width at half-maximum is the coherence time $T_{c}$.

The correlation function $\Gamma^{(1)}(\vec{r}, \tau)$ is the Fourier transform (FT) of the power spectrum $|\widetilde{E}(\vec{r}, \omega)|^{2}, \widetilde{E}$ denoting the FT of the field $E$. Consequently, the (local) spectral bandwidth, defined as the full width at half-maximum of the power spectrum, is inversely proportional to the coherence time. The proportionality factor depends on the shape of the field amplitude. For example, for a Gaussian function, $T_{c} \Delta \nu=0.44$ (with $\Delta \nu=\Delta \omega / 2 \pi$ ). The coherence time is in general shorter than the pulse duration, and equal to it for a 
FT-limited pulse. The measurement of the coherence time in different regions of the spatial profile provides information on the space-dependent spectral content of the harmonics.

Next we consider a field whose temporal dependence can be written as $\exp \left[-i\left(\omega_{0} t+\phi(t)\right)\right]$ (here we neglect the effect of a finite pulse duration on the spectrum, concentrating on the phase variation). The spectral bandwidth is equal to the frequency variation, the so-called chirp, during the pulse given by $\Delta \omega=\Delta d \phi(t) / d t$. The coherence time is thus a measure of the chirp of the radiation at a given point in space.

According to the semiclassical description of harmonic generation, briefly described in Sec. I, a harmonic field can be approximately written as [see Eq. (1)]

$$
E_{q}(\vec{r}, t)=A_{1} \exp \left(-i \alpha_{1} I(\vec{r}, t)\right)+A_{2} \exp \left(-i \alpha_{2} I(\vec{r}, t)\right) .
$$

For the sake of simplicity, we neglect for the moment the temporal variation of the amplitudes of the two contributions. As explained in Ref. [5], the different spatial variation of the phase of the two contributions leads to their spatial separation in the far field. The measurement of the coherence time in the two (inner and outer) spatial regions gives information on the frequency variation of the two components. This frequency variation, given by $\Delta \omega_{j}(t)=\Delta\left[\partial \phi_{j}(t) / \partial t\right]$ $=\Delta \alpha_{j}[\partial I(t) / \partial t]$, presents a common term, namely, the derivative of the fundamental field, and a factor specific to the trajectory: the phase coefficient $\alpha_{j}$. The coherence time is therefore approximately proportional to $1 / \alpha_{j}$. Comparing the coherence times corresponding to different contributions thus gives information on the relative phase coefficients for the corresponding trajectories. The coherence time therefore gives direct information on the fundamental process (the electronic trajectories), leading to the emission of harmonics [10].

In the following we present the predictions of calculations, with different levels of complexity, of the variation of the coherence time (or some parameter proportional to it) for the two main trajectories contributing to harmonic generation as a function of the harmonic order. The underlying assumption of our analysis is that the temporal and spatial separation of the harmonic radiation into two components, and the corresponding coherence times, are closely related to the singleatom dynamics. More specifically they are related to the phase imposed on the harmonic dipole moment by the dynamics of the electron in the continuum. These results are then compared to the experimental data in Sec. III B.

First we use the classical model from Ref. [8] to describe the electronic motion in the continuum. Then we proceed to calculate the dipole moment in a much more sophisticated way, by numerical integration of the time-dependent Schrödinger equation [14]. The third step in our calculations also includes propagation of the generated radiation through the nonlinear medium.

\section{B. Classical model}

Using the classical model introduced in Ref. [8], we omit the influence of the atomic core and consider only the motion of a free electron in a laser field. We also consider a field with a slowly varying amplitude, so that it can be treated as

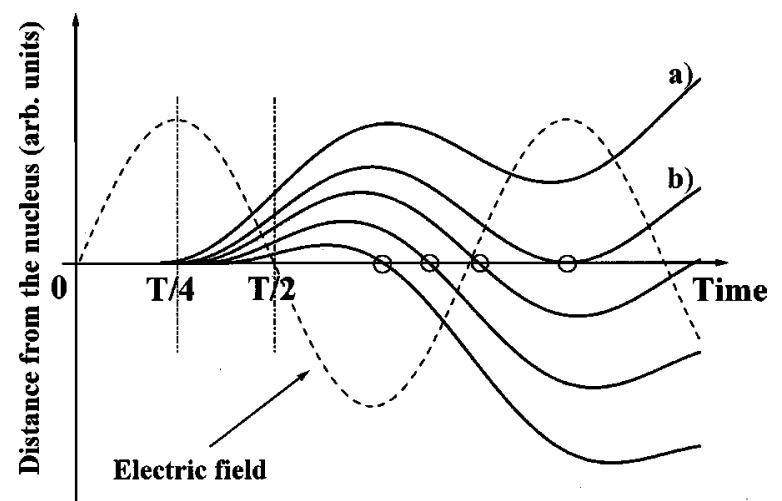

FIG. 3. Schematic representation of the electron trajectories in the continuum, corresponding to different emission times. For the sake of clarity, only the first half period of the laser field is analyzed. Trajectory (a) represents a trajectory where the electron is accelerated away from the core and does not contribute to harmonic generation. Trajectory (b) returns to the core with zero kinetic energy.

approximately constant during a single optical cycle. The force that the electron experiences is proportional to the laser electric field $E=E_{0} \sin (\omega t)$, and the solution of the classical equations of motion for the electron can be written as

$$
\begin{aligned}
\mathrm{v}\left(t, t_{0}\right)= & -\mathrm{v}_{0} \cos (\omega t)+\mathrm{v}_{0} \cos \left(\omega t_{0}\right), \\
x\left(t, t_{0}\right)= & \frac{1}{\omega}\left(-\mathrm{v}_{0} \sin (\omega t)+\mathrm{v}_{0} \sin \left(\omega t_{0}\right)\right) \\
& +\left(t-t_{0}\right) \mathrm{v}_{0} \cos \left(\omega t_{0}\right) .
\end{aligned}
$$

Here $\mathrm{v}_{0}=q E_{0} / m \omega$, and we assume that the electron escapes from the atom at the position $x=0$ at time $t=t_{0}$ with a null initial velocity.

Depending on $t_{0}$, the electron may follow different routes in the continuum. A pictorial representation of the electron trajectories for different emission times is shown in Fig. 3. Examining just the first half optical cycle, it can be easily observed that the electrons emitted while the field is growing in absolute value $(0<t<T / 4)$ are accelerated away from the core and never return to it: such trajectories do not contribute to the process of harmonic generation [trajectory (a)

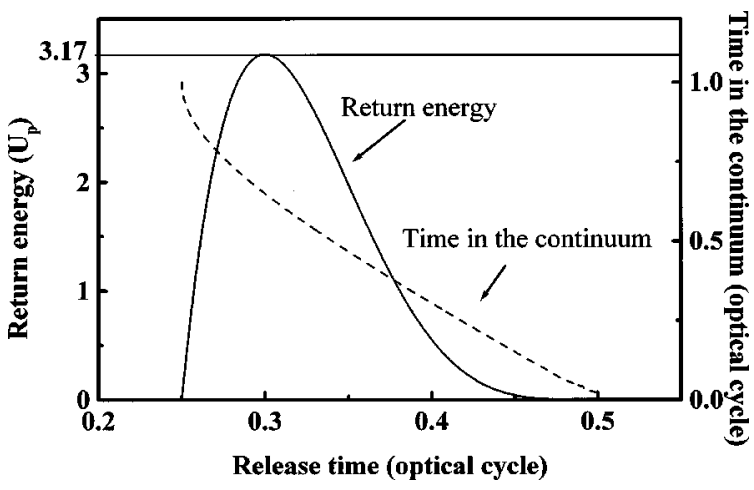

FIG. 4. Return kinetic energies (solid line), expressed in units of the ponderomotive energy, and excursion times $\tau$ (dashed line) of the electrons as a function of their release time $t_{0}$. 


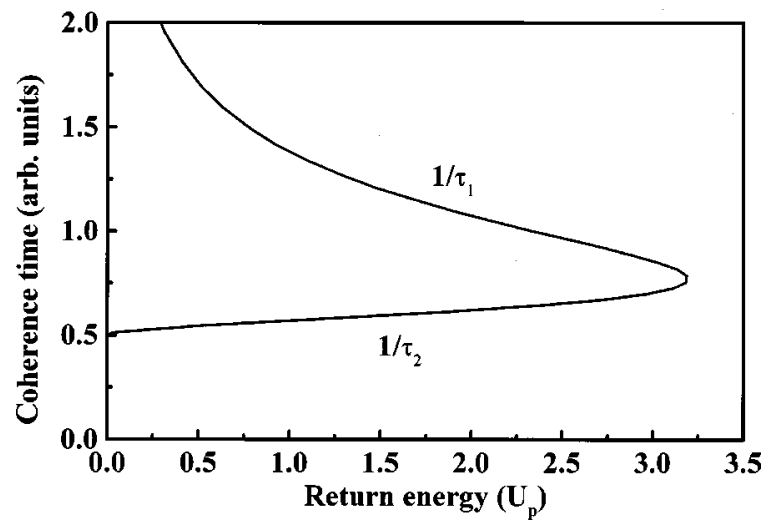

FIG. 5. Coherence times, calculated as the inverse of the excursion times, using a simple classical model, as a function of the ponderomotive energy $\left(U_{p} \propto I \lambda^{2}\right)$.

in the figure]. If the electron escapes at the peak of the field oscillation $(t=T / 4)$, its trajectory brings it back to the core with a zero velocity after a full optical period [trajectory (b) in the figure]. Electrons emitted when the field is decreasing in absolute value $(T / 4<t<T / 2)$ come back and recombine with the core in a shorter time and with higher kinetic energy (the high kinetic energy corresponds to a large slope when crossing the time axis). Finally, electrons which escape into the continuum when the field is close to zero $(t \approx T / 2)$ spend a very short time before recombination, and acquire just a small amount of kinetic energy from the field.

In Fig. 4 we plot the return kinetic energy (expressed in units of the ponderomotive energy, $U_{p} \propto I$ ) and the time $\tau$ spent in the continuum $\left[\tau=t_{r}-t_{0}\right.$, where $t_{r}$ is the recombination time defined by $\left.x\left(t_{r}, t_{0}\right)=0\right]$ as functions of the emission time $t_{0}$.

The kinetic-energy curve presents a well-defined peak corresponding to the maximum energy acquired by electrons during the oscillation in a field of given intensity. This value of the kinetic energy, added to the ionization energy $I_{p}$ of the atom, determines the maximum energy of the emitted photons and corresponds to the well known cutoff law $E_{\text {cutoff }}$ $\approx I_{p}+3.17 U_{p}[8,15]$. Harmonics close to the cutoff come from electrons that escape into the continuum only during a restricted time interval $t \approx 0.3 T$ (see Fig. 4).

On the other hand, for a given electron energy below $3.17 U_{p}$, there are always two possible release times within each half period of the laser field. For relatively low energies, there may be additional, longer, trajectories that bring electrons back to the core, but their contribution to harmonic generation is small, and we do not consider them here.

Harmonics in the plateau are then essentially generated by two different classes of electrons, with two different release times and, correspondingly, different amounts of time spent in the continuum. Electrons released shortly after the peak of the field oscillation have a much longer excursion time compared to those released when the field amplitude is approaching zero. These distinct classes of electrons are responsible for the two components observed for harmonics generated in the plateau.

In Sec. III A we discussed how the coherence time $T_{c j}$ is proportional to $1 / \alpha_{j}$. The phase coefficient $\alpha_{j}$ is closely related to the excursion time $\tau_{j}$, and the variation of $\alpha_{j}$ as

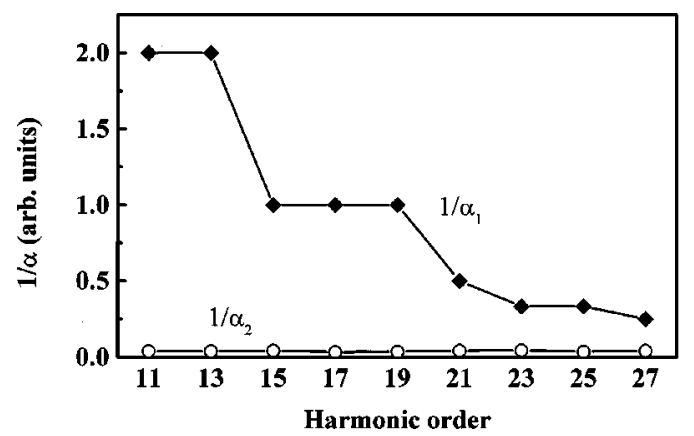

FIG. 6. Coherence times, calculated as the inverse of the phase coefficients $\alpha_{1}$ (corresponding to the short trajectory) and $\alpha_{2}$ (corresponding to the long trajectory), as a function of harmonic order.

function of photon energy follows that of $\tau_{j}$. Therefore, in Fig. 5, we plot the inverse of the two classical excursion times $1 / \tau_{1}$ and $1 / \tau_{2}$, to illustrate the variation of the coherence times with the harmonic order (as well as with the laser intensity). In the figure, we see how the two curves merge when the photon energy (electron return kinetic energy plus ionization potential) increases, becoming identical when the cutoff energy is reached.

\section{Single-atom calculations}

A much more sophisticated description of the electron dynamics is achieved by considering a "real" atom in a strong laser field, and solving the time-dependent Schrödinger equation (TDSE). The calculation is performed in several steps, described in detail in previous papers $[10,14,16]$. Briefly, we start by numerically integrating the TDSE for an argon atom interacting with a short laser pulse, as described in Ref. [14]. The calculation is performed for a laser pulse with a given peak intensity, and the harmonic spectrum is calculated by Fourier transforming the timedependent dipole moment. We then use an adiabatic approximation to extract the intensity dependence of the strength and phase of each harmonic, as described in Ref. [16]. Having obtained the intensity dependence of the dipole moment, $d_{q}(I)$, for each harmonic, we perform a quantum path analysis $[10,17]$ of the harmonic phase, in order to extract the weights of the different paths $\left[A_{j}\right.$; see Eq. (1)] and their corresponding phase coefficients $\left(\alpha_{j}\right)$ as function of the intensity. Briefly, for each intensity $I_{0}$ we multiply the dipole moment with a window function $W_{I_{0}}(I)$ which has its maximum at $I=I_{0}$. The Fourier transform of $d_{q}(I) W_{I_{0}}(I)$ then yields the distribution of reciprocal intensities, or phase coefficients, $\alpha$, at the intensity $I_{0}$ :

$$
A_{I_{0}}(\alpha)=\int d_{q}(I) W_{I_{0}}(I) \exp (i \alpha I) d I .
$$

A first estimate of the coherence times is $T_{c j} \propto 1 / \alpha_{j}$. The results obtained in argon, for a peak intensity of 2 $\times 10^{14} \mathrm{~W} / \mathrm{cm}^{2}$, are presented in Fig. 6. These coherence times agree qualitatively well with those obtained by the classical calculation (Fig. 5).

As explained in Sec. III A, the above approximation for the coherence times considers only the phase variation of the field components, and neglects the effect of a finite pulse 


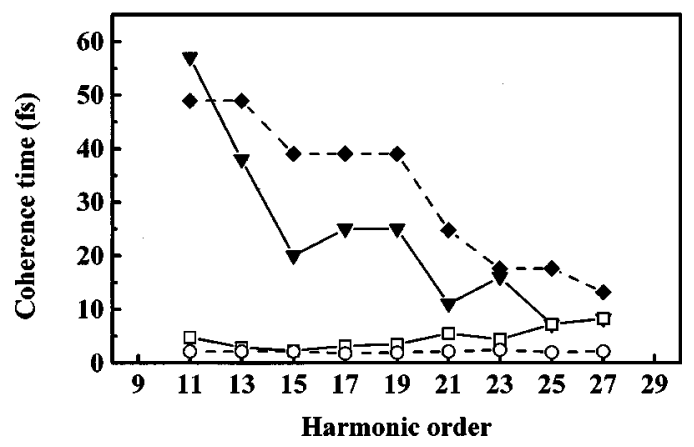

FIG. 7. Coherence times, calculated using the inverse of the phase coefficients, and also accounting for the finite pulse duration (dashed line). Solid diamonds correspond to the short trajectory, and open circles to the long trajectory. Coherence times calculated in a fully macroscopic calculation are drawn with a solid line. Solid triangles correspond to the short trajectory, and open squares to the long trajectory.

duration. This is valid when the induced chirp is dominant, which is not the case for the component with the long coherence time (top curve in Figs. 5 and 6). For this component, the spectral broadening induced by the finite duration of the harmonic pulse (the Fourier-transform limited bandwidth) is comparable to the bandwidth induced by the dipole chirp. To account for the finite pulse duration in a simple and approximate way [18], we assume a Gaussian variation for the amplitude of the harmonic field and a linear frequency chirp, $b_{j}$, proportional to the phase coefficient $\alpha_{j}$. The time dependence of each component of the harmonic field can be written as $E_{j}(t)=\exp \left(-a_{H} t^{2}-i\left(\omega_{0} t-b_{j} t^{2}\right)\right)$, where $a_{H}$ $=2 \ln 2 / T_{H}^{2}$, and $T_{H}$ is the harmonic pulse duration. For such a field, the coherence time is shorter than the harmonic pulse duration by the factor $\sqrt{1+\left(b_{j} / a_{H}\right)^{2}}$. In Fig. 7, we show the coherence times calculated in the same conditions as in Fig. 6 , but accounting for the finite-pulse duration of the harmonics (dashed lines). We assume here that all the harmonics have a pulse length equal to half that of the fundamental. As expected, the longest coherence times are affected the most by the finite pulse duration.

\section{Calculations including propagation}

Finally, we perform a complete calculation including propagation, and thereby phase matching, of the harmonic field through the nonlinear medium. We use the single-atom dipole moment - calculated as a function of the intensity — as a source term of the nonlinear part of the polarization field, and solve the wave equation in the paraxial and slowly varying envelope approximations by numerical propagation through the medium. This method was described extensively, for instance, in Ref. $[16,19]$. We then calculate the harmonic spectrum at the exit of the medium, by Fourier transforming the (space-dependent) harmonic time profile. From the spectrum, which presents a superposition of two curves with different widths [10], we obtain the bandwidth and hence the coherence time of each of the two components. Note that in this calculation very few approximations have been made. We automatically take into account the small intensity dependency of the phase coefficients, since we are using the full dipole moment as a source term in the macroscopic cal-

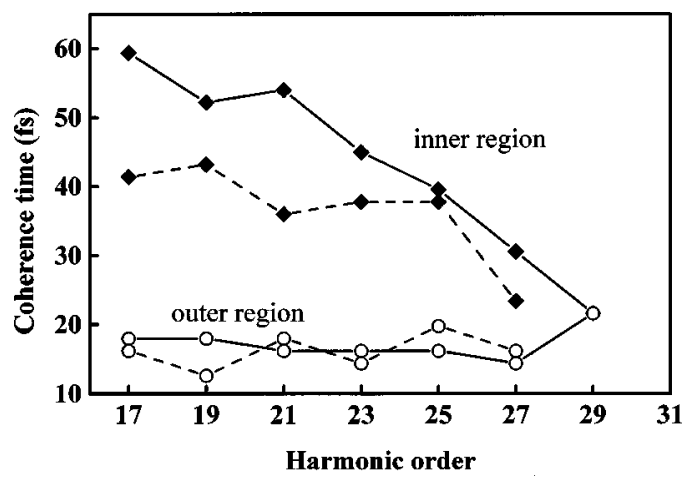

FIG. 8. Coherence times measured experimentally as a function of harmonic order. The dashed lines correspond to an intensity of $2 \times 10^{14} \mathrm{~W} / \mathrm{cm}^{2}$ and the solid lines to an intensity of 4 $\times 10^{14} \mathrm{~W} / \mathrm{cm}^{2}$. The inner region is marked with diamonds and the outer region with circles.

culation. The results are presented in Fig. 7 (solid lines). The behavior of the coherence times is quite close to that shown with dashed lines. This leads to the conclusion that the coherence times are essentially governed by the simple physics contained in the classical model, with the correction that the effect of a finite pulse duration must be accounted for.

\section{EXPERIMENTAL RESULTS}

We measure the variation of the coherence times of the two regions for different harmonic orders for two different intensities, $2 \times 10^{14} \mathrm{~W} / \mathrm{cm}^{2}$ (dashed lines in Fig. 8) and 4 $\times 10^{14} \mathrm{~W} / \mathrm{cm}^{2}$ (solid lines in Fig. 8). We observe two regions with different coherence times: an outer region with a short coherence time (open circles), and an inner region with a longer coherence time (solid diamonds). The coherence time for the outer region is approximately constant with respect to both harmonic order and intensity, whereas the coherence time for the inner region decreases with harmonic order and increases with intensity. The dependence on the harmonic order is very similar to the theoretical predictions (Fig. 7). There is some difference regarding the absolute values for the coherence times (Figs. 7 and 8), especially for the outer region but the trends are the same. Let us emphasize again that the aim of our measurements is not to determine accurate coherence times, but to study variations and trends.

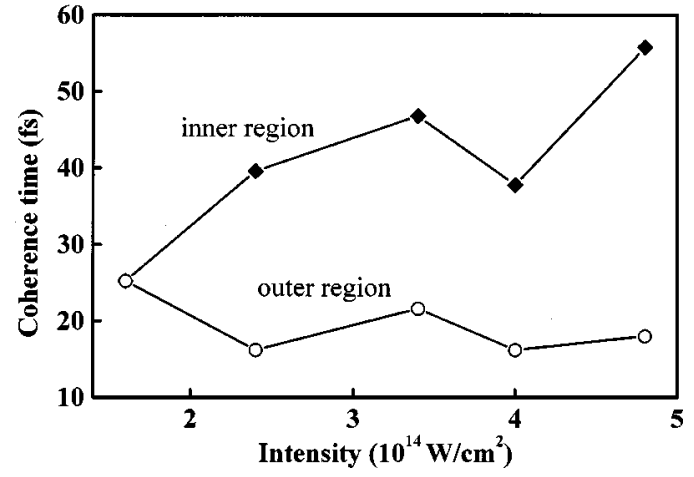

FIG. 9. Coherence times measured experimentally as a function of intensity for the 23rd harmonic. The inner region is represented by diamonds, and the outer region by circles. 


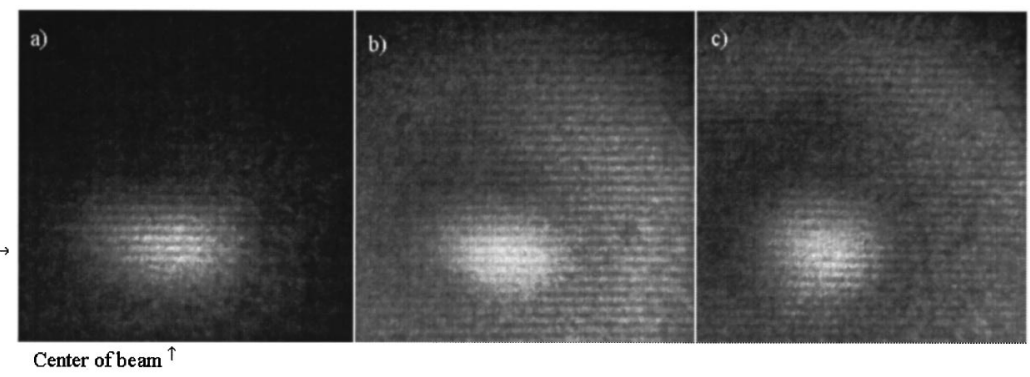

FIG. 10. Interference pattern for different focus positions for the 13th harmonic: (a) the focus is $2.4 \mathrm{~mm}$ before the gas jet; (b) the focus is in the center of the gas jet; and (c) the focus is 1.8 $\mathrm{mm}$ after the gas jet.

The difference between theory and experiment might simply be due to some systematic error owing to our method of determining the experimental coherence times.

The behavior of the coherence time with respect to intensity is presented in Fig. 9, where the coherence time for the 23rd harmonic is plotted for increasing intensities. The coherence time of the inner region increases with increasing intensity, whereas the outer region remains relatively insensitive. An increase of the intensity corresponds to moving harmonics in the plateau region away from the cutoff. According to our theoretical understanding presented above, the two trajectories and hence the two coherence times should therefore differ more and more as the intensity is increased. This agrees with our experimental observations.

In Fig. 10, the influence of the focus position relative to the gas jet is illustrated. We record the interference pattern while moving the position of the focus through the gas jet for the 13th harmonic. The beam is apertured to a diameter of 6 $\mathrm{mm}$, and the peak intensity is estimated to be 3 $\times 10^{14} \mathrm{~W} / \mathrm{cm}^{2}$. We see how the relative importance of the two regions varies for the different conditions. In (a), where the focus is before the gas jet, the outer region has disappeared and harmonics are emitted only in the inner region. In (b) and (c), where the focus is positioned in the gas jet, and after the gas jet, respectively, both regions can be seen. This figure illustrates clearly how phase-matching conditions influence the contributions of the two trajectories differently. When the focus is before the gas jet, we see only the contribution due to the shortest trajectory (with excursion time $\left.\tau_{1}\right)$. The contribution of the other, longer, trajectory is not properly phase matched. This agrees well with theoretical calculations presented in Ref. [10]. As the focus is moved through the gas jet, the contribution of the other trajectory (with excursion time $\tau_{2}$ ) is better phase matched and the outer region becomes visible. In (c) the outer region is slightly annular, which is consistent with previous measurements of the spatial profile of the harmonic radiation [20].
We also find that the coherence times in the two regions do not depend on the focus position. This is because they are essentially determined by the single-atom dynamics, and not by propagation effects.

\section{CONCLUSION}

We have studied the coherence times of high-order harmonics produced in argon. The spatial profiles in the far field separate into two distinct spatial regions having different coherence times, in agreement with previous results. We have measured the coherence times of the inner and outer regions as function of the harmonic order, the laser intensity, and the position of the gas jet.

The coherence times vary both as a function of the harmonic order, and as a function of the laser intensity, in good agreement with the predictions of the semiclassical model. In this model, the two dominating quantum paths contributing to the generation of some particular harmonic are distinct when the harmonic is deep in the plateau region, and become more alike as the harmonic approaches the cutoff region. We observe this effect on the coherence times, which directly reflect the electronic trajectories. This is a clear experimental signature of the semiclassical model. The fact that we can enhance one or the other of the two regions by macroscopically changing the focusing conditions is in good agreement with predictions taking into account phase matching of the two components of the field in the nonlinear medium $[10,21]$.

\section{ACKNOWLEDGMENTS}

We would like to thank K.C. Kulander and K.J. Schafer for the use of their single-atom data. We acknowledge the support of the Swedish Natural Science Research Council and the EC "Access to Large Scale Facilities" Program (Contract No. ERBFMGECT950020).
[1] A. Bouhal, R. Evans, G. Grillon, A. Mysyrowicz, P. Breger, P. Agostini, R. C. Constantinescu, H. G. Muller, and D. von der Linde, J. Opt. Soc. Am. B 14, 950 (1997).

[2] T. E. Glover, R. W. Schoenlein, A. H. Chin, and C. V. Shank, Phys. Rev. Lett. 76, 2468 (1996).

[3] T. Ditmire, E. T. Grumbell, R. A. Smith, J. W. G. Tisch, D. D. Meyerhofer, and M. H. R. Hutchinson, Phys. Rev. Lett. 77, 4756 (1996).

[4] See, for example, R. Haight and D. R. Peale, Phys. Rev. Lett. 70, 3979 (1993); J. Larsson, E. Mevel, R. Zerne, A. L'Huillier,
C.-G. Wahlström, and S. Svanberg, J. Phys. B 28, L53 (1995); M. Gisselbrecht, D. Descamps, C. Lyngå, A. L'Huillier, C.-G. Wahlström, and M. Meyer, Phys. Rev. Lett. 82, 4607 (1999).

[5] M. Bellini, C. Lyngå, A. Tozzi, M. B. Gaarde, T. W. Hänsch, A. L'Huillier, and C.-G. Wahlström, Phys. Rev. Lett. 81, 297 (1998).

[6] R. Zerne, C. Altucci, M. Bellini, M. B. Gaarde, T. W. Hänsch, A. L'Huillier, C. Lyngå, and C.-G. Wahlström, Phys. Rev. Lett. 79, 1006 (1997).

[7] K. J. Schafer, B. Yang, L. F. DiMauro, and K. C. Kulander, 
Phys. Rev. Lett. 70, 1599 (1993).

[8] P. B. Corkum, Phys. Rev. Lett. 71, 1994 (1993).

[9] M. Lewenstein, Ph. Balcou, M. Yu Ivanov, A. L'Huillier, and P. B. Corkum, Phys. Rev. A 49, 2117 (1994).

[10] M. B. Gaarde, F. Salin, E. Constant, Ph. Balcou, K. J. Schafer, K. C. Kulander, and A. L'Huillier, Phys. Rev. A 59, 1367 (1999).

[11] M. Lewenstein, P. Salières, and A. L'Huillier, Phys. Rev. A 52, 4747 (1995).

[12] S. Svanberg, J. Larsson, A. Persson, and C.-G. Wahlström, Phys. Scr. 49, 187 (1994).

[13] O. Svelto, Principles of Lasers (Plenum Press, New York, 1993).

[14] K. J. Schafer and K. C. Kulander, Phys. Rev. Lett. 78, 638 (1997)

[15] J. L. Krause, K. J. Schafer, and K. C. Kulander, Phys. Rev.
Lett. 68, 3535 (1992).

[16] M. B. Gaarde, Ph. Antoine, A. L'Huillier, K. J. Schafer, and K. C. Kulander, Phys. Rev. A 57, 4553 (1998).

[17] Ph. Balcou, A. S. Dederichs, M. B. Gaarde, and A. L'Huillier, J. Phys. B 32, 2973 (1999).

[18] A. E. Siegman, Lasers (University Science Books, Mill Valley, CA, 1986).

[19] Ph. Antoine, D. B. Milosević, A. L'Huillier, M. B. Gaarde, P. Salières, and M. Lewenstein, Phys. Rev. A 56, 4960 (1997).

[20] P. Salières, T. Ditmire, K. S. Budil, M. D. Perry, and A. L'Huillier, J. Phys. B 27, L217 (1994); see also P. Saliéres, A. L'Huillier, and M. Lewenstein, Phys. Rev. Lett. 74, 3776 (1995).

[21] Ph. Antoine, A. L'Huillier, and M. Lewenstein, Phys. Rev. Lett. 77, 1234 (1996). 